\title{
Dynamic properties of additively manufactured tantalum
}

\author{
Matthew Cotton ${ }^{1 *}$, and Beth Girling ${ }^{1}$ \\ ${ }^{1}$ AWE, Aldermaston, Reading, Berkshire, RG7 4PR, UK.
}

\begin{abstract}
In this study a series of gas gun plate impact experiments have been conducted on samples of wire arc AM tantalum to investigate the high strain rate behaviour of the material, including tensile failure (spall) mechanisms. Conventionally processed tantalum was fielded alongside the AM material to provide a direct comparison under identical loading conditions. In-situ velocimetry data was supported by pre-shock characterisation of the samples to supply information on the initial material microstructure, and to link these features to the observed differences in dynamic behaviour. Additional post-shock analysis of the damaged region in the samples provided further insight into the failure process.
\end{abstract}

\section{Introduction}

Recent years have seen rapid developments in the capabilities of additive manufacturing (AM), changes which have the potential to significantly increase the applications for AM components. AM is an attractive manufacturing route due to its ability to produce complex shapes with minimal material wastage, and the flexibility of its CAD based design-to-build methodology. As AM technology has matured, there has been a drive to extend the range of materials which can be processed by AM routes. In the field of metallic AM, steel, titanium and aluminium alloys been the subject of most research due to the ability to produce largely defect free material at $\sim 100 \%$ density. Other materials have proven to be less amenable to AM, often due to high melting temperature which makes laser powder bed fusion methods less effective.

One such material is tantalum, a high density refractory metal with a melting point of $3017 \mathrm{~K}$. In this study, tantalum samples were produced using a wire arc AM (WAAM) process [1], in which a wire feedstock is melted by an electron beam heat source. The use of this technique makes it possible to achieve the temperatures necessary to fully melt the Ta wire over the short timescales required to make it viable for manufacturing part-scale components [2]. This process takes place on a moveable scanning head which allows the molten material to be deposited on the build platform to build a component in a layerwise fashion. Although WAAM lacks the ability of powder bed fusion to produce small scale features, it is particularly well-suited for the manufacture of large volume parts.

\footnotetext{
* Corresponding author: matthew.cotton@awe.co.uk
} 
In order to increase confidence in the use of AM components in practical applications, it is important to evaluate the constitutive properties of an AM material with respect to its conventional equivalent. A common observation of AM metals is the novel, highly anisotropic microstructure which is produced by the high thermal gradients which are established during the build process. This has been shown to influence the mechanical properties of the material [3-4], and can affect the ability to qualify a component for use. Another factor of particular relevance to AM materials is the presence of process-induced defects [5-6] which can provide preferential locations for early initiation of failure mechanisms, and is one of the main barriers to wider use of AM components.

In order to ascertain the high strain rate behaviour of WAAM Ta, a series of gas gun plate impact experiments have been conducted to evaluate the nucleation of damage and onset of spall. AM samples were fielded alongside conventional wrought Ta samples to provide a direct comparison between the two under identical loading conditions. Samples were diagnosed using optical velocimetry to provide velocity-time profiles, from which spall strength was determined. Pre- and post-experiment characterisation of the sample provided information on the connection between initial material microstructure and the internal damage nucleation and growth caused by the state of dynamic tensile stress induced in the material.

\section{Material}

In order to evaluate the capabilities of the WAAM technique for use with Ta a hemispherical shell was manufactured, building outwards from a conventional Ta rod at the pole. The completed hemishell is shown in figure 1.

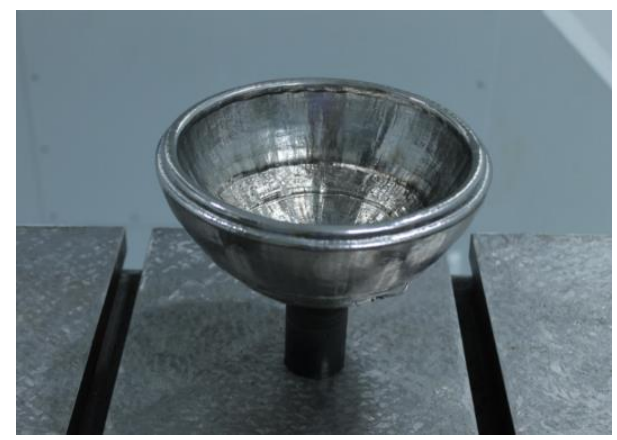

Fig. 1. WAAM Ta hemishell with conventional Ta rod at pole.

Samples for these experiments, in the form of $30 \times 3 \mathrm{~mm}$ discs, were cut from the shell at an angle of $20^{\circ}$ from the equator. The conventional Ta samples used to perform a comparison with the WAAM material was taken from the rod.

Sound speed measurements were taken from both WAAM and conventional samples using ultrasonic transducers to introduce longitudinal and shear waves and recording the transit time through the sample thickness. These values can then be used to determine the bulk sound speed $\left(\mathrm{C}_{\mathrm{B}}\right)$ using equation 1 .

$$
C_{B}=\sqrt{C_{L}^{2}-\frac{4}{3} C_{S}^{2}}
$$


Measured and calculated wave speeds for the two samples are shown in table 1.

Table 1. Sound speeds in conventional and WAAM Ta.

\begin{tabular}{|c|c|c|c|}
\hline & $\mathrm{C}_{\mathrm{L}}(\mathrm{mm} / \mu \mathrm{s})$ & $\mathrm{C}_{\mathrm{S}}(\mathrm{mm} / \mu \mathrm{s})$ & $\mathrm{C}_{\mathrm{B}}(\mathrm{mm} / \mu \mathrm{s})$ \\
\hline Conventional Ta & 4.153 & 2.101 & 3.370 \\
\hline WAAM Ta & 4.320 & 2.033 & 3.627 \\
\hline
\end{tabular}

The WAAM material returned a faster $\mathrm{C}_{\mathrm{L}}$ and slower $\mathrm{C}_{\mathrm{S}}$ when compared to conventional $\mathrm{Ta}$, a result which may be related to the high degree of orientation in the WAAM microstructure [7]. Longitudinal and shear waves oscillate in orthogonal planes, so the effect of propagating through a highly anisotropic environment may well be to speed up one and slow the other as observed here. The changes result in a small increase in the calculated WAAM Ta bulk wave speed, a value which can be compared to the $\mathrm{C}_{0}$ term in the experimentally derived Hugoniot.

Prior to testing the density of each sample was calculated from the measured dimensions and mass. This provides some information on the quality of the WAAM material, as errors in the build process can lead to the formation of porosity which is reflected in reduced density. The average value recorded for the eight samples used in this series is $16.42+/-0.095 \mathrm{~g} / \mathrm{cc}$, or $98.62 \%$ when expressed in terms of relative density. This is slightly below the values which have been achieved using AM with metals such as steel or Ti-6Al-4V which have been the subject of more extensive technique development, but is high enough to give confidence that there is no significant porosity.

EBSD images were taken to supply information on grain size, orientation and aspect ratio, factors which are significantly affected by the material processing route. Comparisons of images from conventional and WAAM Ta are shown in figure 2.
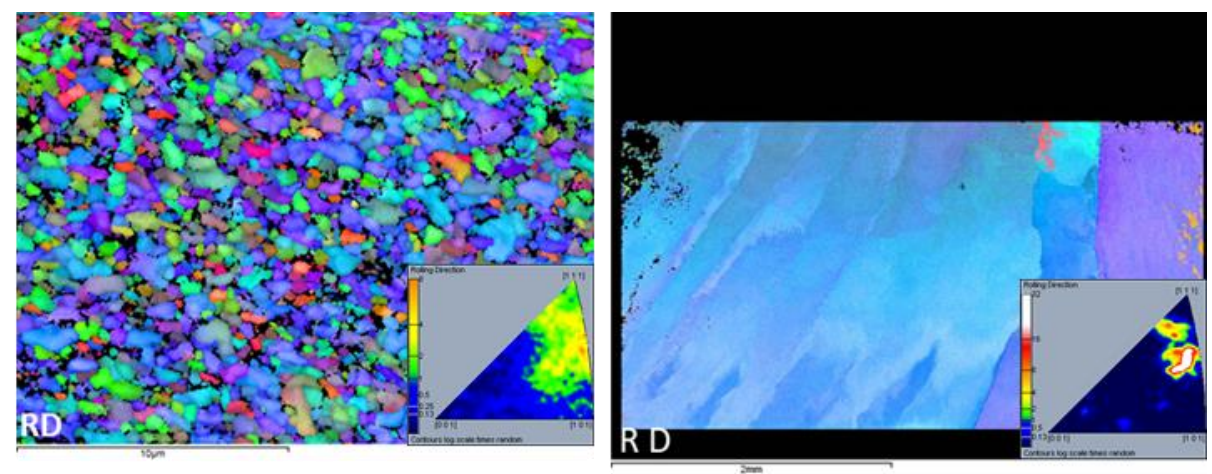

Fig. 2. EBSD image of conventional Ta (l) and WAAM Ta (r), viewed along rolling direction.

The conventional material displays an equiaxed grain structure with no strong preferred orientation. In contrast the WAAM Ta has a structure characterised by large columnar grains of several $\mathrm{mm}$ in length along the axis of the grain. Inverse pole figures from the sample region indicate preferred orientations of $>20 \mathrm{x}$ random, with these orientations not always aligning with the principal crystallographic axes. The grain morphology observed in the WAAM Ta is caused by the epitaxial growth of grains along the direction of the large thermal 
gradients induced in the material by the rapid heating and cooling which occurs during the WAAM process. The angle of the grain growth with respect to the sample axis is due to the mismatch between the vertical build direction and the orientation of the sample disc within the hemishell wall at $20^{\circ}$ from the equator.

\section{Results}

Experiments were conducted using a $70 \mathrm{~mm}$ bore single stage gas gun, with impact velocities in the range $167-762 \mathrm{~m} / \mathrm{s}$. Both WAAM and conventional samples were fielded side-byside in the target assembly. All shots used a copper impactor with the exception of shot 6 , which used tungsten heavy alloy to generate a higher stress in the target.

The principal diagnostic fielded on these trials was heterodyne velocimetry (HetV), recording from the rear surface of each Ta target. The final shot in the series did not field any rear surface HetV diagnostics to allow the target to spall unimpeded. HetV data from the other shots are presented in composite form in figure 3. Jump off times have been aligned to aid comparison.
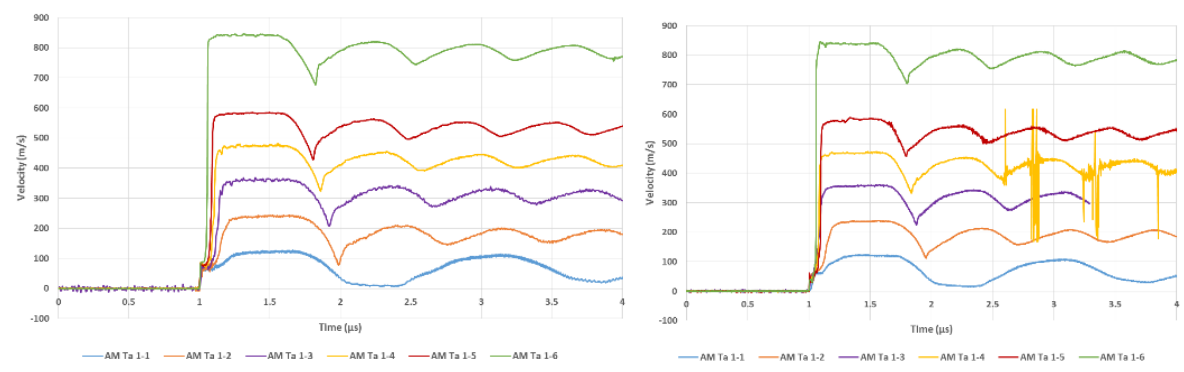

Fig. 3. HetV data recorded from conventional Ta samples (l) and WAAM Ta samples (r).

\section{Analysis}

Data from the HetV spectrograms was processed to generate points on the principal Hugoniot of the targets. These values have been plotted in figure 4 as individual data points in the $U_{S^{-}}$ $\mathrm{u}_{\mathrm{p}}$ plane, alongside a line representing the linear $\mathrm{U}_{\mathrm{S}}-\mathrm{u}_{\mathrm{p}}$ relationship for conventional Ta taken from Marsh [8].

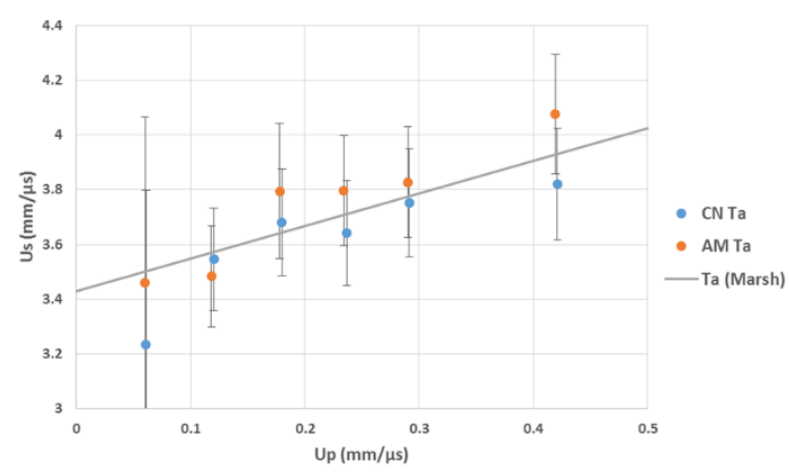

Fig. 4. Us- $\mathrm{u}_{\mathrm{p}}$ Hugoniot data from this series, compared to existing Ta data. 
The data from this series is in good agreement with the existing Ta Hugoniot, a result which accords with previous investigations of AM metals under shock conditions [9-10]. For every shot except shot 2, the WAAM Ta sample returned a slightly higher shock velocity than the conventional Ta sample, a finding which is consistent with the higher calculated wave speed from the static sound speed measurements. Excluding shot 2, the average increase in shock velocity for the WAAM Ta was $0.17 \mathrm{~mm} / \mu \mathrm{s}$, in reasonable agreement with the increase of $0.26 \mathrm{~mm} / \mu \mathrm{s}$ seen in the value of $\mathrm{C}_{\mathrm{B}}$.

As discussed above, the HetV traces from shots 2-6 display the characteristic rapid reload which is indicative of spall in the targets. The magnitude of the recorded velocity pullback is proportional to the spall strength of the material, and these values have been calculated using equation 2 .

$$
\sigma_{s p}=0.5 \rho_{0} C_{0}\left(\Delta u_{f s}+\delta\right), \delta=\left(\frac{h_{s p}}{c_{0}}-\frac{h_{s p}}{C_{L}}\right) \cdot\left|u_{1}\right|
$$

$\rho_{0}=$ initial density

$\mathrm{C}_{0}=$ bulk sound speed

$\mathrm{C}_{\mathrm{L}}=$ longitudinal sound speed

$\Delta \mathrm{u}_{\mathrm{fs}}=$ velocity pullback magnitude

$\mathrm{h}_{\mathrm{sp}}=$ spall layer thickness

$\mathrm{u}_{1}=$ velocity pullback gradient

Using equation 2, and adjusting the sound speed values for the WAAM Ta based on the measurements taken for this work, spall strengths have been calculated for the Ta samples.

Measured values of spall strength were found to be consistently higher in the conventional Ta, with an average value of $6.52 \mathrm{GPa}$ compared to $5.22 \mathrm{GPa}$ in the WAAM Ta. Post-shot, the targets were cut in half to expose the spall plane and examine the extent of the void nucleation and crack growth. The overall void volume and distribution was broadly comparable between targets on the same shot, contained within a band of $\sim 0.5 \mathrm{~mm}$ thickness in the centre of the target.

The fully spalled targets from shot 7 were separated to expose the spall surface, from which a series of SEM images were gathered. This supplied further insight into the mechanism of tensile failure in the two samples. Images from the two samples are shown in figure 5 .
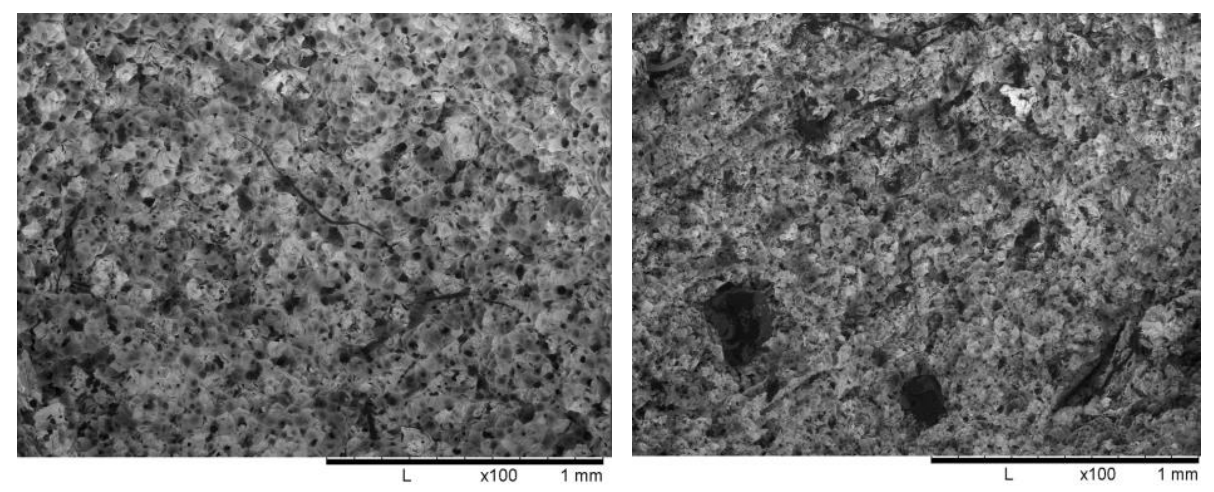

Fig. 5. Conventional Ta spall surface (1) and WAAM Ta spall surface (r). 
Images of the spall surface indicate that the morphology of the failure plane is similar, with both samples showing signs of ductile dimples of approximately the same size. However, some differences between the samples are apparent, with larger voids distributed across the WAAM Ta surface which are not seen in the conventional material. These voids are likely to be caused by manufacturing defects such as incomplete melting or entrapped gas, and are reflected in the slight reduction in density of the WAAM Ta relative to conventional Ta. When the samples are placed in tension, these voids will act as preferential locations for the initiation and growth of spall damage, leading to the observed reduction in spall strength in the WAAM Ta.

\section{Conclusions}

A series of gas gun plate impact experiments have been conducted to investigate the dynamic behaviour of WAAM Ta, and to evaluate the extent of any differences from conventional Ta. Observations from this investigation include the highly anisotropic WAAM microstructure, the small variation seen along the material Hugoniot, and a $\sim 20 \%$ reduction in spall strength in the WAAM Ta. These results highlight the potential for even limited manufacturing defects to influence the failure mechanisms in WAAM material, and the requirement to fully characterise material produced by AM routes alongside testing under relevant loading conditions.

The authors would like to extend their thanks to Andrew Johnson for manufacture of the WAAM Ta, Glenn Whiteman and Zach Gower for their help in conducting the experiments, and to Neil Bond and Chris Poulter for analysis of the samples.

CBritish Crown Owned Copyright 2021/AWE

\section{References}

1. S. W. Williams, F. Martina, A. C. Addison, J. Ding, G. Pardal, P. Colegrove, Mater. Sci. Technol. 32641 (2016).

2. G. Marnielli, F. Martina, S. Ganguly, S. Williams, Int. J. Refract. Met. Hard. Mater. 83 104974 (2019).

3. B. E. Carroll, T. A. Palmer, A. M. Beese, Acta Mat. 87309 (2015).

4. B. Song, E. Nishida, B. Sanborn, M. Maguire, D. Adams, J. Carroll, J. Wise, B. Reedlunn, J. Bishop, T. Palmer, J. Dynamic Behavior Mater. 3412 (2017).

5. X. Zhao, S. Li, M. Zhang, Y. Liu, T. B. Sercombe, S. Wang, Y. Hao, R. Yang, L. E. Murr, Materials and Design 9521 (2016).

6. T. Ronneberg, C. M. Davies, P.A. Hooper, Materials and Design 189108481 (2020).

7. G. Marnielli, F. Martina, S. Ganguly, S. Williams, Additive Manufacturing 32101009 (2020).

8. S. P. Marsh (Ed.), LASL Shock Hugoniot Data, University of California Press (1980).

9. G. T. Gray, V. Livescu, P. A. Rigg, C. P. Trujillo, C. M. Cady, S. R. Chen, J. S. Carpenter, T. J. Lienert, S. J. Fensin, Acta. Mat. 138140 (2017).

10. D. R. Jones, S. J. Fensin, O. Dippo, R. A. Beal, V. Livescu, D. T. Martinez, C. P. Trujillo, J. N. Florando, M. Kumar, G. T. Gray, J. Appl. Phys. 120135902 (2016). 